\title{
Frequency Dependent CV Measurements of GaAs/AlGaAs Heterostructures
}

M. Berroth, R. Bosch and V. Hurm

Fraunhofer Institut fuer Angewandte Festkoerperphysik

Eckerstr. 4, 7800 Freiburg, FRG

Summary

A procedure is described to determine the carrier density profile in the channel of a FET by evaluating S-parameters measured over a broad frequency range. Applying this method to GaAs/AlGaAs heterostructures, a frequency dispersion of the gate capacitance has been found, which is attributed to a parasitic conducting channel.

Introduction

The capacitance measurement of a Schottky contact versus the applied bias voltage is a commonly used technique to determine the carrier concentration profile of a device. Due to the operating frequency of about $1 \mathrm{MHz}$, which is very low compared to applications of field effect transistors far in the $\mathrm{GHz}$ range, relatively large structures are required, so that the carrier profile can not be measured at the transistors used in microwave circuits. Further the junction resistance becomes rapidly smaller than the impedance of the junction capacitance at low frequencies, if an increasing forward bias is applied thus making $\mathrm{CV}$ measurements difficult. In addition, some of these transisors like MESFETs and MODFETs are showing low frequency effects; $\mathrm{CV}$ measurement at high frequencies would therefore be of great interest.

Measurements

The measurements are performed using a HP 8510 network analyzer with a Cascade prober for on-wafer testing in a $50 \mathrm{ohms}$ environment. Different samples have been measured using a standard microwave transistor with 1 
gate length and $250 \mu \mathrm{m}$ width. The drain to source voltage is set to zero and the scattering parameter $S_{11}$ is mensured at various gate voltages from beyond pinch-off up to far positive gate voltages. The S-parameters are then converted to the $\mathrm{Z}$-parameters to simplify the deembedding.

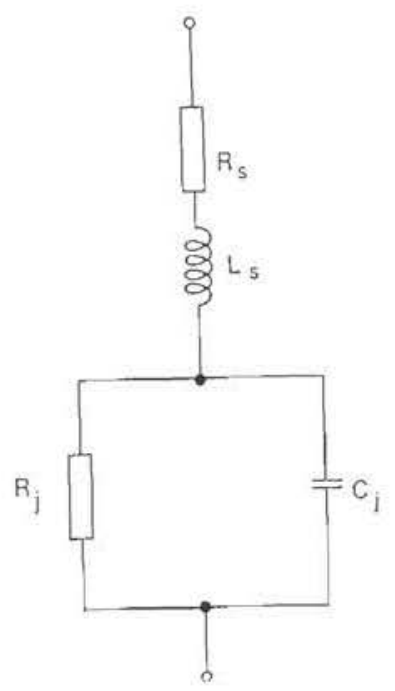

Fig. 1: Equivalent circuit of a Schottky contact

Deembedding Procedure

As shown in fig. 1, the equivalent circuit of a Schottky contact at high frequencies consists of four elements: a parasistic olmmic resistance in series with an inductance and the intrinsic Schottky contact, described as a junction resistance and a junction capacitance in parallel. The impedance of the Schottky contact is given by the following equation:

$$
Z_{11}=R_{s}+j \omega L_{s}+\frac{R_{j}}{1+j \omega R_{j} C_{j}}
$$

Assuming that the parasitic ohmic resistance and inductance are independent of the bias, both can be determined precisely if the gate is strongly positive biased, because at these high current levels the junction resistance is negligeable and shunts the junction capacitance. The real part of the Z-parameters describes the ohmic series resistance, while the imaginary part determines 
the inductance at strongly positive gate voltage. Using this procedure to determine the parasitic series resistance and inductance, the measured $\mathrm{Z}$ parameters at all bias points and frequencies can now simply be deembedded by subtracting $R$ s from the real part and $\omega L_{s}$ from the imaginary part of $Z_{11}$. The resultant $Z$-parameters are then converted to $Y$-parameters, the real part of which represents the juntion conductance, and the imaginary part the junction capacitance.

\begin{tabular}{|c|c|c|c|c|}
\hline $800 \AA$ & GaAs & $G$ & doped & $3 \cdot 10^{18} \mathrm{~cm}^{-3}$ \\
\hline $50 \AA$ & AlGias & weth storis & doped & $x^{18} \mathrm{men}^{-3}$ \\
\hline $300 \AA$ & GaAs & & doped & 1.70 con \\
\hline $400 \AA$ & GaAs & & ${ }^{\text {undoped }} 2$ & $D E G^{\text {Spacel }}$ \\
\hline $50 \AA$ & AlGaAs & & thanped & \\
\hline $400 \AA$ & AlGins & & doped & $1.10^{18} \mathrm{~cm}^{-3}$ \\
\hline $2000 \AA$ & AlGaAs & & undoped & buffer \\
\hline
\end{tabular}

Fig. 2: Cross-sectional view of the hetcrostructure transistor

Results

We have measured MESFETs grown by MBE on an AlGaAs buffer layer. The CV curves determined were very close to those measured at $1 \mathrm{MHz}$ for the whole range from $50 \mathrm{MHz}$ up to $25 \mathrm{GHz}$. However a modulation doped ficld effect transistor with a vertical structure, as shown in fig. 2 , exhibited a strong frequency dependence of the junction capacitance, as presented in fig. 3. The proposed explanation for this behaviour is that there is a parasitic conductive channel in the doped AlGaAs layer. The diminishing of this path at higher frequencies is thought to be due to the increased contact resistance 
to this layer and the reduced mobility of the carriers, establishing a low pass response.

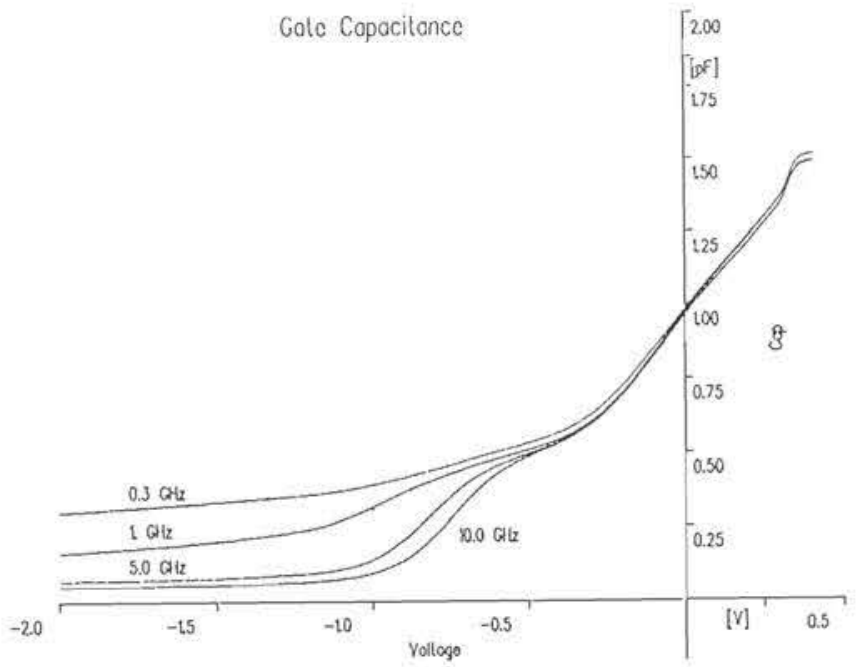

Fig. 3: Gate capacitance extracted from S-parameter data for various freguencies

Conclusion

A method to determine the gate capacitance of even submicrometer gate transistors is proposed. Due to the broad band S-parameter measurement this procedure is of great interest for the characterization of heterostructure FETs, e.g. GaAs/AlGaAs MODFETs. Unlike other methods (1), no fitting procedure is necessary. Especially for the characterization of thin layers needed in cnhancement FETs can this procedure be used to determine the junction capacitance of a Schottky contact up to moderately positive bias voltages.

\section{Refercnces}

1. Y. K. Fukai and M. Muraguchi, "New Method for Measuring Carrier Concentration Profile Near a GaAs Surface Through a Network Analyzer," IEEE Electron Device Letters, vol. 9, pp. 74-76, Feb, 1988. 